\title{
Standardization Specification and Effect Size, the Two Major Limitations of Clinical Trials of Herbal Medicine
}

\author{
Ahmad Shamabadi ${ }^{a, b}$ \\ ${ }^{a}$ School of Medicine, Tehran University of Medical Sciences, Tehran, Iran; b Psychiatric Research Center, Roozbeh \\ Psychiatric Hospital, Tehran University of Medical Sciences, Tehran, Iran
}

Dear Editor,

Herbal medicine has attracted the attention of researchers in the prevention and treatment of diseases due to evidence of its effectiveness, probable safety, cost-benefit analysis acceptability, and public acceptance. However, for scientific consumption, herbs need to be evaluated like approved drugs in the long-established scientific process - from understanding the possible mechanisms to phase II and III clinical trials [1]. Clinical trials have been conducted for a long time, but there are significant limitations to many of them, two of which this paper aims to address.

Observance of purity standards for herbal products is one of the limitations. In many trials, the plant name is written, but the standardization is not specified. DNA barcoding of 44 plant products in one study by Newmaster et al. detected significant substitution, contamination, and fillers and suggested the poor quality of most of them [2]. Apart from the importance of the purity of one herb, different herbals may be used in studies that provide their intervention herbals from the market [3]. The actual example was using Hypericum perforatum preparations artificially enriched in hyperforin for marketing reasons that interacted with selective serotonin reuptake inhibitors [4]. In other words, in addition to finding the main valuable ingredients, it will be possible to find the culprit in issues of adverse events with a proper characterization.
Although randomized controlled trials are the gold standard for evaluating effectiveness, obtaining a significant $p$ value through them does not necessarily mean clinical effectiveness. Even a small effect can cause statistical significance, while this effect size cannot be detected and distinguished in the clinic [5]. In many clinical trials, including herbal ones and not restricted to them, the word "effectiveness" is used after reaching a significant difference with the control group, while this effect will not be detectable in the clinic and means superiority to placebo.

This paper seeks to conclude that the propensity for herbal medicines should not lead to immature judgments about their prescription and use. The results of clinical studies without proper characterization and without obtaining clinical significance will not help recommend the prescription. Based on the evidence of effectiveness obtained from previous trials, studies on herbals should be continued, but limitations should be considered in conclusions and rectified as much as possible. Importantly, characterization of plants materials, i.e., origin, which plant part, quantification of a lead substance or active ingredient, if available, the extraction solvent, and the drugextract ratio should be done.

\section{Conflict of Interest Statement}

The author has no conflicts of interest to declare. 


\section{Funding Sources}

This paper did not receive any specific grant from funding agencies in the public, commercial, or not-for-profit sectors.

\section{Author Contributions}

A.S. wrote this comment.

\section{References}

1 Eder J, Herrling PL. Trends in modern drug discovery. New Approaches to Drug Discovery; 2015. pp. 3-22.

2 Newmaster SG, Grguric M, Shanmughanandhan D, Ramalingam S, Ragupathy S. DNA barcoding detects contamination and substitution in North American herbal products. BMC Med. 2013 Oct;11(1):222.

3 Kahkeshani N, Hadjiakhoondi A, Khanavi M. Differentiation of Lavandula angustifolia
Mill. and Nepeta menthoides Boiss \& Buhse using microscopic and phytochemical evaluations. 2014.

4 Davis SA, Feldman SR, Taylor SL. Use of St. John's Wort in potentially dangerous combinations. J Altern Complement Med. 2014 Jul;20(7):578-9.

5 Wasserstein RL, Lazar NA. The ASA statement on p-values: context, process, and purpose. Taylor \& Francis; 2016. 